\title{
Anti-Hyperalgesic Effects of Meloxicam Hydrogel via Phonophoresis in Acute Inflammation in Rats; Comparing Systemic and Topical Application
}

\author{
Tae-Youl KIm ${ }^{1}$, Young-II KIm ${ }^{2}$, Sam-Ki SEO ${ }^{3}$, Soo-Hyeun KIm ${ }^{1}$, Kyu-Ho YANG ${ }^{4}$, and Sang-Chul SHIN ${ }^{5, *}$ \\ ${ }^{1}$ Department of Physical Therapy, Dongshin University, Naju 520-714, ${ }^{2}$ Department of Pharmaceutical Engineering, \\ Konyang University, Nonsan 320-711, ${ }^{3}$ Department of Physical Therapy, Graduate School, Seonam University, \\ Namwon 590-711, ${ }^{4}$ School of Dentistry, Dental Research Institute and Second Stage BK 21, Gwangju 500-757, \\ ${ }^{5}$ College of Pharmacy, Chonnam National University, Gwangju 500-757, Republic of Korea
}

(Received June 23, 2009; Revised July 11, 2009; Accepted July 13, 2009)

\begin{abstract}
The aim of this study was to determine if a meloxicam hydrogel could be administered in vivo via phonophoretic transdermal delivery using pulsed ultrasound by examining its anti-hyperalgesic effects in a rat carrageenan inflammation model. Carrageenan (1\%) was injected into the plantar surface of the right hindpaw, and meloxicam hydrogel was administered via phonophoretic transdermal delivery. Changes in the mechanical and thermal hyperalgesia, as well as swelling, showed that phonophoretic delivery of meloxicam exhibited significantly better anti-hyperalgesic and anti-inflammatory effects than pulsed ultrasound. Topical and systemic application of meloxicam hydrogel using phonophoresis showed similar anti-hyperalgesic effects. These findings suggest that the transdermal administration of a meloxicam hydrogel using phonophoresis by pulsed ultrasound might be useful for treating acute inflammation.
\end{abstract}

Keywords: Meloxicam hydrogel, Phonophoretic transdermal delivery, Anti-hyperalgesia, Systemic application, Topical application, Acute inflammation

\section{INTRODUCTION}

Meloxicam, a non-steroidal anti-inflammatory drugs (NSAIDs) with both anti-inflammatory and anti-noceptive actions, is widely used in clinical practice (Todd and Sorkin, 1988; Mishra and Vijaya Kumar, 2006). Delivering NSAIDs through the skin for either local or systemic treatment has been investigated (Rolf et al., 1999; Cagnie et al., 2003). Local and systemic effects that can be distinguished by determining the local tissue drug concentrations and plasma levels (Cagnie et al., 2003).

The skin provides potential for non-invasive drug delivery (Foldvari, 2000). However, the low permeability of skin due to the barrier properties of the stratum corneum limits the passive transdermal approach of most drugs (Median et al., 1995; Fang et al., 2001). Many studies have focused on methods for overcoming the barrier properties of the stratum corneum and for promoting drug permeability using chemical agents or physical modalities (Jadoul et al., 1999).

*Corresponding author

Tel: +82-62-530-2924 Fax: +82-62-530-2949

E-mail: shinsc@chonnam.ac.kr
Phonophoresis (Tyle and Agrawala, 1989), which uses ultrasound with a chemical agent, is often applied for transdermal delivery in clinics with iontophoresis using direct current (Tyle and Agrawala, 1989; Riviere and Heit, 1997). Phonophoresis has been used successfully to deliver anti-inflammatory medication to inflamed subcutaneous tissue (Bare et al., 1996; Cagnie et al., 2003; Yang et al., 2005; Yang et al., 2008). The major advantages are the introduction of medication to a local area without invasion of the skin and the synergistic interaction of ultrasound and drugs (Wells, 1977). Phonophoresis with NSAIDs is commonly used to treat inflamed tissues (Byl, 1995; Hsieh, 2006). The direct peripheral anti-hyperalgesic effects of ultrasound and phonophoresis with NSAIDs in treating injured tissue have been widely investigated (Guffey and Knaust, 1997), but the possible effects of ultrasound and phonophoresis with NSAIDs on the central modulation of inflammatory nociception are still unclear (Hsieh, 2006).

This study examined the effectiveness of meloxicam using phonophoretic transdermal delivery by determining its anti-hyperalgesic effects in a rat carrageenan inflammation model. We compared the anti-hyperalgesia and anti-edema effects of meloxicam hydrogel, as well as differences in top- 
ical and systemic administration.

\section{MATERIALS AND METHODS}

\section{Animals}

Male Sprague-Dawley rats $(253 \pm 1.23 \mathrm{~g})$ were purchased from the Daehan Laboratory Animal Research Co. (Choongbug, Korea), and given access to normal standard chow diet (Jae II Chow, Korea) and tap water ad libitum. The animals were housed in laminar flow cages, four or five rats to a cage, and were maintained at $22 \pm 1^{\circ} \mathrm{C}$, relative humidity of $40-60 \%$, and a $12-\mathrm{h}$ light-dark cycle. The animals were allowed to acclimatize for at least one week before the experiments. The experiments were performed in accordance with the "ethical standards set by the Helsinki Declaration of the World Medical Association" in 1964, revised in 2004.

\section{Preparation of meloxicam hydrogel}

One gram of carbopol 940 was dissolved in $40 \mathrm{ml}$ of distilled water. Then, $20 \mathrm{ml}$ of propylene glycol, $5 \mathrm{ml}$ of Labrafil and $30 \mathrm{ml}$ of ethanol containing meloxicam was mixed with constant stirring and adjusted to $\mathrm{pH} 7.0$ using triethanolamine. Water was added to the solution to make a final volume of $100 \mathrm{ml}$. The prepared gels were stable at $50^{\circ} \mathrm{C}$ for 6 months.

\section{Induction of hyperalgesia}

Paw hyperalgesia was induced by injecting $1 \%$ carrageenan as described by Levy (1969) and Schrier et al. (1987). Prior to drug treatment, a $1 \%$ carrageenan suspension $(0.1 \mathrm{ml})$ was injected into the subplantar area of the right hindpaw and the withdrawal latency was measured. The animals were tested before injecting carrageenan (Dowdall et al., 2005) as the baseline (time 0), and at 3, 6, 9 $\mathrm{h}$ after the carrageenan injection. The animals were returned to their cage until they were tested.

\section{Paw swelling test}

The extent of paw swelling was determined by measuring the change in the paw volume. The paw swelling was measured on the right foot using a digimatic caliper (Mitytoyo, Japan), before and at 3,6 , and $9 \mathrm{~h}$ after carrageenan injection. Their mean values were calculated (the average of three determinations per group).

\section{Mechanical pain threshold}

For the measurement of mechanical pain threshold, rats were placed in transparent lucite cubicles that allow minimal movement on an elevated meshed platform. The von Frey monofilaments (Touch - Test Sensory Evaluation, North Coast Medical, USA) were applied were applied at right angles to the plantar surface of the paw starting with the lowest bending force and then increasing with logarithmically incremental stiffness $(1.4,2,4,6,8,10,15,26 \mathrm{~g})$ to calculate the $50 \%$ probability thresholds for mechanical paw withdrawal. Mechanical pain threshold, i.e., the lowest bending force at which the animal lifts its paw off the meshed platform, was noted. Their mean values were calculated (the average of three determinations per group).

\section{Thermal pain threshold}

To determine the paw withdrawal latency, animals were placed on a hot plate (DJM, Korea) with a surface temperature of $30^{\circ} \mathrm{C}$ for 10 minutes. The surface temperature of the hot plate was increased to $50 \pm 0.2^{\circ} \mathrm{C}$ and the paw withdrawal latency time was determined. The time for paw withdrawal was counted from the time the heat was applied to the subplantar area of the right hindpaw showing hyperalgesia. Any lifting associated with normal locomotion was excluded. The lifting of the right paw could be explained by a combination of factors including thermal exposure and the animal adjusting its weight onto the uninjured paw (Dowdall et al., 2005). Their mean values were calculated (the average of three determinations per group).

\section{Drug application}

Primary experiment: The changes in anti-hyperalgesia and anti-swelling effects by dose of meloxicam were determined. The rats were divided into four groups of six each. The group I (control group) did not receive any treatment. The meloxicam hydrogel $(0.5 \%)$ was used on the abdomen for group II, $1 \%$ for group III, and $2 \%$ for group IV.

Secondary experiment: We then compared the effects of topical and systemic meloxicam hydrogel. The rats were divided into five groups of six each. The group I (control group) did not receive any treatment. The meloxicam hydrogel $(1 \%)$ with phonophoresis was used on the abdomen area in group II or on the paw area in the group III. In groups II and III, an ultrasound transducer with $0.8 \mathrm{~cm}^{2}$ of effective radiation area (ERA) and 6.0 max beam non-uniformity ratio (BNR) which indicates the relationship between the spatial peak intensity and spatial average intensity (Bare et al., 1996). The treatment parameter was a pulsed mode, its duty cycle was $20 \%$, the ultrasound frequency was $1 \mathrm{MHz}$, the treatment intensity was $1.0 \mathrm{~W} / \mathrm{cm}^{2}$, the treatment area was two-fold wider than the ERA, and the treatment time was 5 min. In groups II and III, approximately $500 \mathrm{mg}$ of the meloxicam hydrogel was applied to the abdomen area and the subplantar area of the right hindpaw. Each group was treat- 
ed once after carrageenan injection.

\section{Statistics}

All values are reported as a mean \pm standard error of five determinations. The data were analyzed and the difference in the variables between the groups and the measuring periods was examined using one way analysis of variance (ANOVA) and Tukey post hoc tests. A $p$ value $<0.05$ was considered significant.

\section{RESULTS}

\section{Primary experiment}

Meloxicam hydrogel decreased paw swelling, indicating anti-hyperalgesia at 3,6 , and $9 \mathrm{~h}(p<0.001)$, but no difference between the $1 \%$ and $2 \%$ meloxicam hydrogel groups (Fig. 1). Meloxicam hydrogel also improved the mechanical pain threshold after $3(p<0.05), 6$, and $9 \mathrm{~h}(p<0.001)$, but no difference between the $1 \%$ and $2 \%$ meloxicam hydrogel

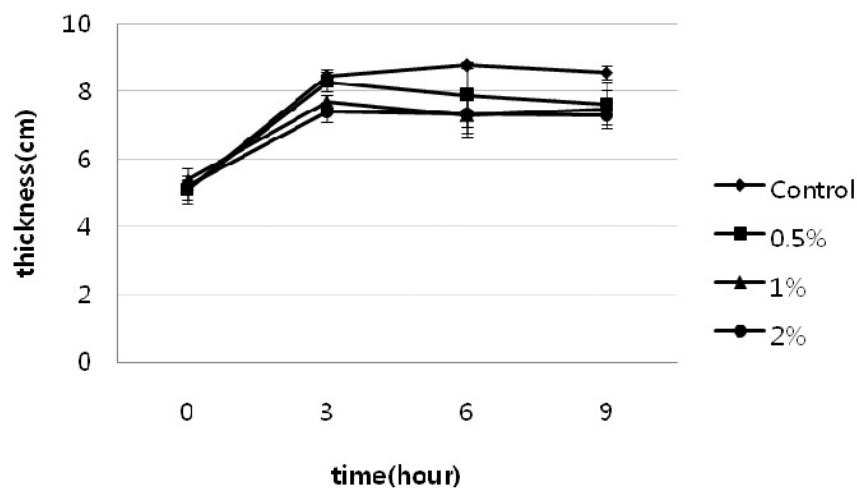

Fig. 1. Effect of the application dose of meloxicam hydrogel on paw swelling. The error bar represents the standard deviation of the mean $(n=3)$.

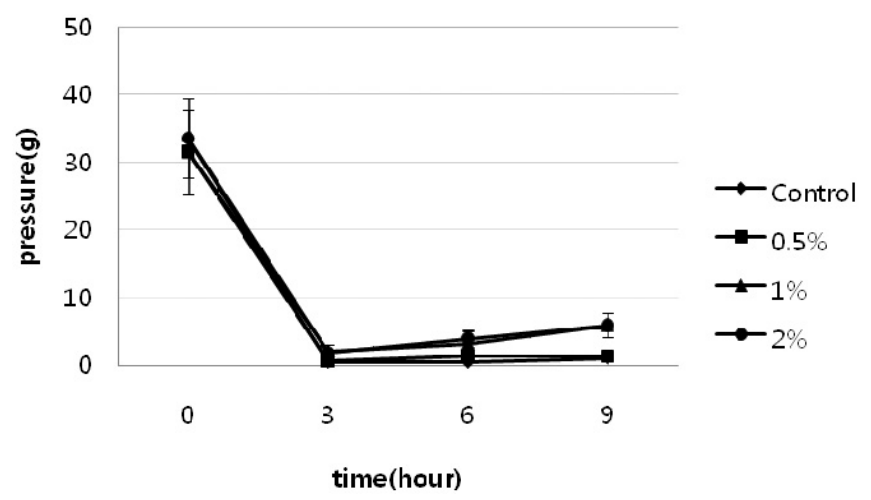

Fig. 2. Effect of the application dose of meloxicam hydrogel on paw mechanical pain threshold. The error bar represents the standard deviation of the mean $(n=3)$. groups (Fig. 2). Similarly, meloxicam hydrogel improved the thermal pain threshold after $3(p<0.05), 6$, and 9 h $(p<$ 0.001 ), with no difference between the $1 \%$ and $2 \%$ meloxicam hydrogel groups (Fig. 3). Therefore, $1 \%$ meloxicam gel was used at secondary experiment.

\section{Secondary experiment}

Meloxicam hydrogel delivered by phonophoresis decreased paw swelling after 3 and $6 \mathrm{~h}$ of application $(p<$ 0.01) (Fig. 4), with no difference in paw or abdomen application. Meloxicam hydrogel phonophoresis also improved the mechanical pain threshold after $3(p<0.05), 6$, and $9 \mathrm{~h}$ $(p<0.001)$ (Fig. 5). Meloxicam hydrogel phonophoresis decreased the thermal pain threshold after 3,6 , and $9 \mathrm{~h}(p<$ 0.001 ) (Fig. 6). Meloxicam hydrogel phonophoresis showed anti-inflammatory effects in the early inflammatory stage, with no differences between paw and abdomen application.

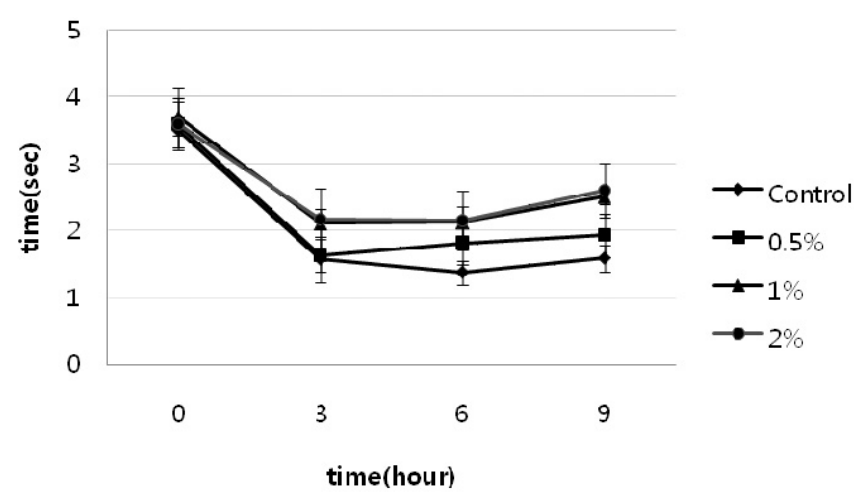

Fig. 3. Effect of the application dose of meloxicam hydrogel on paw thermal pain threshold. The error bar represents the standard deviation of the mean $(n=3)$.

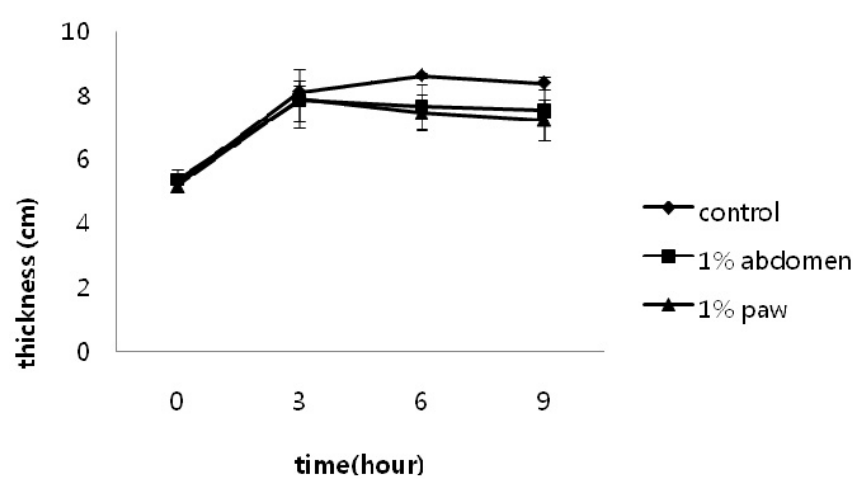

Fig. 4. Effect of the application site of meloxicam hydrogel on paw swelling. The error bar represents the standard deviation of the mean $(n=6)$. 


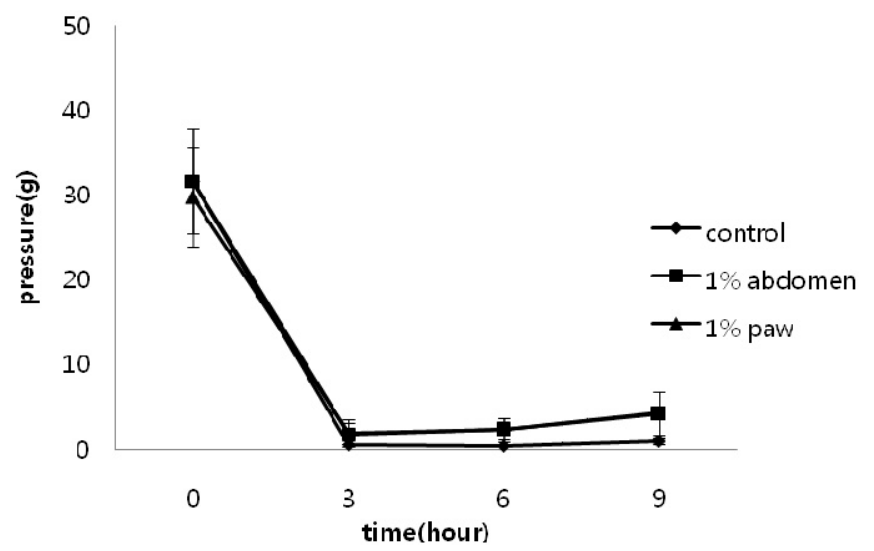

Fig. 5. Effect of the application site of meloxicam hydrogel on paw mechanical pain threshold. The error bar represents the standard deviation of the mean $(n=6)$.

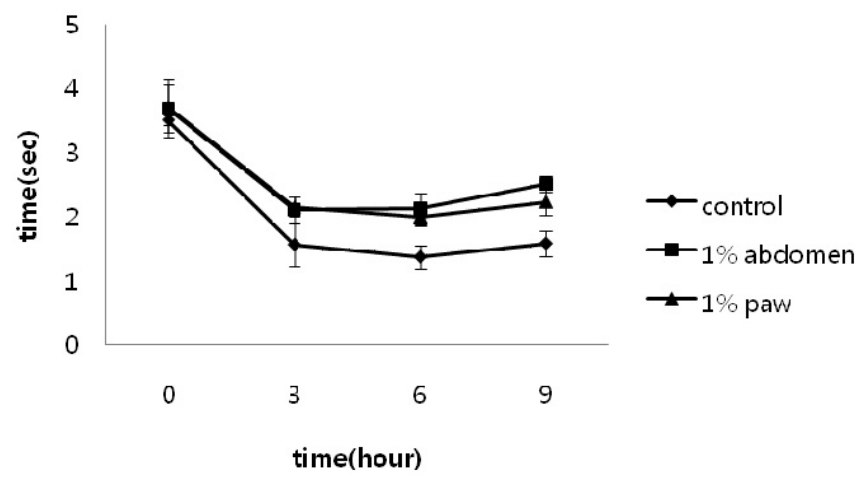

Fig. 6. Effect of the application site of meloxicam hydrogel on paw thermal pain threshold. The error bar represents the standard deviation of the mean $(n=6)$.

\section{DISCUSSION}

We examined the possibility of using phonophoretic administration of meloxicam clinically by determining its antihyperalgesic effects in a rat carrageenan inflammation model. Carrageenan (1\%) was injected into the plantar surface of the right hindpaw, and we measured the anti-edema and anti-hyperalgesic effects of meloxicam after phonophoretic transdermal delivery systemically or topically. Meloxicam activity was not different in the $1 \%$ and $2 \%$ groups (Fig.1-3). Cui et al. (2008) reported no difference in 1, 3, or $5 \%$ meloxicam patches, and NSAID dose did not influence pain control in osteoarthritis patients (Yocum et al., 2000).

NSAIDs do not change pain thresholds in acute inflammation (Sekiguchi et al., 2008) suggesting that drug dose does not influence anti-hyperalgesic and anti-edema effects. Meloxicam hydrogel phonophoresis improved the mechanical and thermal pain threshold 3 and $6 \mathrm{~h}$ after car- rageenan injection, as well as anti-hyperalgesic effects (Fig. $5,6)$.

NSAIDs inhibit the synthesis of prostaglandins from arachidonic acid by inhibiting cyclo-oxygenase (COX) activity (Vane, 1971; Needleman et al., 1986). COX-1 is involved in maintaining physiologic functions such as gastric protection and homeostasis, whereas COX-2 is involved in pathophysiologic process such as inflammation, pain, and fever (Gajraj, 2003). Meloxicam is a selective COX-2 inhibitor (Xin et al., 2007), which mediates its anti-hyperalgesic effect (Abramson and Weissmann, 1989; Francischi et al., 2002). Nociceptors are sensitized whenever the major COX-like prostaglandins are isolated from the inflammatory sites (Cesare and McNaughton, 1997; Chen et al., 1999), which cause hyperalgesia (Ferreira, 1980). Meloxicam hydrogel produced similar or better effects than anti-inflammatory drugs using other administration routes (Dirig et al., 1998; Jett et al., 1999; Feltenstein et al., 2004).

Meloxicam hydrogel phonophoresis reduced paw swelling from 3 to $6 \mathrm{~h}$ after carrageenan injection, demonstrating increased anti-inflammatory effects in the early inflammatory stage, with no difference in paw or abdomen application (Fig. 4). Phonophoresis application reduced paw swelling more than ultrasound and showed similar effects to systemic application. Phonophoresis application also improved mechanical and thermal pain thresholds better than the other groups (Fig. 5,6). The inhibition of COX reduces the sensitization of peripheral afferents and therefore alleviates the hyperalgesia associated with tissue inflammation (Herrero et al., 1997).

In many studies on phonophoresis, continuous ultrasound was used to enhance the delivery of anti-inflammatory drugs (Ciccone et al., 1991; Kozanoglu et al., 2003). Ultrasound waves are associated with nonthermal and mechanical effects such as vibration, pressure, and cavitation of cells as the waves pass through the tissue (Nyborg, 1985). The thermal effects can be reduced by using a pulsed mode of ultrasound, and these mechanical effects may facilitate drug permeation by vibrating the molecules in the tissue and drug media, altering the cell membrane and increasing cell permeability (Bommannan et al., 1992).

NSAIDs are effective analgesics in situations of hyperalgesia related to tissue inflammation (Herrero et al., 1997). The anti-inflammatory action of NSAIDs was caused by the suppression of the prostaglandin synthesis pathways by inhibiting the COX enzyme (Vane, 1971; Lim et al., 2008). NSAIDs have similar anti-inflammatory effects in carrageenan or complete Freund adjuvant (CFA) induced inflammation (Hsieh, 2006). Iontophoresis of piroxicam (Penzes 
et al., 2005) and diclofenac sodium (Gao et al., 2002), phonophoresis of diclofenac sodium (Rosim et al., 2005; Vlak, 1999), and phonophoresis of meloxicam gel (Choi et al., 2006) could treat the musculoskeletal inflammatory disorders using common drugs by transdermal route. NSAIDs are used to treat inflammatory diseases of the musculoskeletal system such as arthritis, tendinitis and bursitis, where physical modalities are less effective.

Romsing et al. (2001) reported that pain control is similar $4 \mathrm{~h}$ after topical or injection. Topical application of NSAIDs has a systemic effect caused by systemic blood circulation (Carter et al., 1997), and ketoprofen concentrations in tissue and blood were similar after phonophoretic application (Cagnie et al., 2003). We found similar results after topical and systemic administration of meloxicam hydrogel by phonophoretic delivery, indicating that phonophoresis provides significant systemic delivery.

\section{CONCLUSIONS}

Phonophoretic transdermal delivery of a meloxicam hydrogel using pulsed ultrasound might be useful for treating acute inflammation via systemic or topical application.

\section{ACKNOWLEDGMENTS}

This study was supported by a grant of the New Technology R\&D Project, Gwangju Technopark (2007).

\section{REFERENCES}

Abramson, S. B. and Weissmann, G. (1989). The mechanisms of action of nonsteroidal anti-inflammatory drugs. Arthritis. Rheum. 32, 1-9.

Bare, A. C., Mcanaw, M. B., Pritchard, A. E., Struebing, J. G., Smutok, M. A., Christie, D. S., Domenech, M. A., Bare, M. A., Bloodworth, M. L. and Seal, L. A. (1996). Phonophoretic delivery of $10 \%$ hydrocortisone through the epidermis of humans as determined by serum cortisol concentrations. Phys. Ther. 76, 738-745; discussion 746-749.

Bommannan, D., Okuyama, H., Staufer, P. and Guy, R. H. (1992). Sonophoresis, I: the use of high frequency ultrasound to enhance transdermal drug delivery. Pharm. Res. 9, 559-564.

Byl, N. N. (1995). The use of ultrasound as an enhancer for transcutaneous drug delivery: phonophoresis. Phys. Ther. 75, 539-553.

Cagnie, B., Vinck, E., Rimbaut, S. and Vanderstraeten, G. (2003). Phonophoresis versus topical application of ketoprofen: comparison between tissue and plasma levels. Phys. Ther. 83, 707-712.

Carter, R., Ebner, D., Brenner, D. and Bruppacher, R. (1997). Use of topical NSAIDs in patients receiving systemic NSAID treatment: A pharmacy-based study in Germany. J. Clinical. Epidemiology. 50, 217-218.
Cesare, P. and Mcnaughton, P. (1997). Peripheral pain mechanisms. Curr. Opin. Neurobiol. 7, 493-499.

Chen, X., Tanner, K. and Levine, J. D. (1999). Mechanical sensitization of cutaneous C-fiber nociceptors by prostaglandin E2 in the rat. Neurosci. Lett. 267, 105-108.

Choi, S. J., Yoon, S. W., Jung, D. I., Kim, Y. I., Jeong, J. G. and Kim, T. Y. (2006). Skin permeation effects of meloxicam gel on ultrasound parameters by phonophoresis. J. Korean Acad. Clin. Electrophysiology. 4, 49-61.

Ciccone, C. D., Leggin, B. G. and Callamaro, J. (1991). Effects of ultrasound and trolamine salicylate phonophoresis on delayed-onset muscle soreness. Phys. Ther. 71, 666-678.

Cui, L., Hou, X., Jiang, J., Li, G., Liang, Y. and Xin, X. (2008). Comparative enhancing effects of electrical treatment with chemical enhancers on transdermal delivery of meloxicam in vitro. pp. 12-15. Institute of Physics Publishing, London.

Dirig, D. M., Isakson, P. C. and Yaksh, T. L. (1998). Effect of COX-1 and COX-2 inhibition on induction and maintenance of carrageenan-evoked thermal hyperalgesia in rats. $J$. Pharmacol. Exp. Ther. 285, 1031-1038.

Dowdall, T., Robinson, I., and Meert, T. F. (2005). Comparison of five different rat models of peripheral nerve injury. Pharmacol. Biochem. Behav. 80, 93-108.

Fang, J. Y., Fang, C. L., Hong, C. T., Chen, H. Y., Lin, T. Y. and Wei, H. M. (2001). Capsaicin and nonivamide as novel skin permeation enhancers for indometacin. Eur. J. Pharm. Sci. 12, 195-203.

Feltenstein, M. W., Schuhly, W., Warnick, J. E., Fischer, N. H. and Sufka, K. J. (2004). Anti-inflammatory and anti- hyperalgesic effects of sesquiterpene lactones from Magnolia and Bear's foot. Pharmacol. Biochem. Behav. 79, 299-302.

Ferreira, S. H. (1980). Peripheral analgesia: mechanism of the analgesic action of aspirin-like drugs and opiate-antagonists. Br. J. Clin. Pharmacol. 10(Suppl 2), 237S-245S.

Foldvari, M. (2000). Non-invasive administration of drugs through the skin: challenges in delivery system design. Pharm. Sci. Technol. 3, 417-425.

Francischi, J. N., Chaves, C. T., Moura, A. C., Lima, A. S., Rocha, O. A., Ferreira-Alves, D. L. and Bakhle, Y. S. (2002). Selective inhibitors of cyclo-oxygenase-2 (COX-2) induce hypoalgesia in a rat paw model of inflammation. $\mathrm{Br}$. $\mathrm{J}$. Pharmacol. 137, 837-844.

Gajraj, N. M. (2003). Cyclooxygenase-2 inhibitors. Anesth. Analg. 96, 1720-1738.

Gao, J. Q., Wang, H., Liang, W. Q. and Chen, M. X. (2002). Transdermal delivery of diclofenac sodium gel after iontophoresis. Zhejiang Da Xue Xue Bao Yi Xue Ban 31, 437-439.

Guffey, J. S. and Knaust, M. L. (1997). The use and efficacy of ultrasound. Rehab. Manag. 10, 44, 48-50.

Herrero, J. F., Parrado, A. and Cervero, F. (1997). Central and peripheral actions of the NSAID ketoprofen on spinal cord nociceptive reflexes. Neuropharmacology. 36, 1425-1431.

Hsieh, Y. L. (2006). Effects of ultrasound and diclofenac phonophoresis on inflammatory pain relief: suppression of inducible nitric oxide synthesis in arthritic rats. Phys. Ther. 86, 39-49.

Jadoul, A., Bouwstra, J. and Preat, V. (1999). Effects of iontophoresis and electroporation on the stratum corneum Review of the biophysical studies. Advanced Drug Delivery 
Reviews. Adv. Drug. Deliv. Rev. 35, 89-105.

Jett, M. F., Ramesha, C. S., Brown, C. D., Chiu, S., Emmett, C., Voronin, T., Sun, T., O'yang, C., Hunter, J. C., Eglen, R. M. and Johnson, R. M. (1999). Characterization of the analgesic and anti-inflammatory activities of ketorolac and its enantiomers in the rat. J. Pharmacol. Exp. Ther. 288, 12881297.

Kozanoglu, E., Basaran, S., Guzel, R. and Guler-Uysal, F. (2003). Short term efficacy of ibuprofen phonophoresis versus continuous ultrasound therapy in knee osteoarthritis. Swiss. Med. Wkly. 133, 333-338.

Levy, L. (1969). Carrageenan paw edema in the mouse. Life Sci. 8, 601-606.

Lim, E. J., Kang, H. J., Jung, H. J., Kim, K. H.. Lim, C. J. and Park, E. H. (2008). Anti-inflammatory, anti-angiogenic and anti-nociceptive activities of 4-hydroxybenzaldehyde. Biomol. Therap. 16, 231-236.

Median, V. M., Walmsley, A. D. and Irwin, W. J. (1995). Phonophoresis - is it a reality? Int. J. Pharm. 118, 129-149.

Mishra, D. N. and Vijaya Kumar, S. G. (2006). Investigations on analgesic, anti-inflammatory and ulcerogenic potential of meloxicam solid dispersion prepared with skimmed milk. Yakugaku Zasshi. 126, 495-498.

Needleman, P., Turk, J., Jakschik, B. A., Morrison, A. R. and Lefkowith, J. B. (1986). Arachidonic acid metabolism. Annu. Rev. Biochem. 55, 69-102.

Nyborg, W. L. (1985). Mechanisms. In Biological Effects of Ultrasound (W. L. Nyborg and M. C. Ziskin, Eds.), pp. 23-33. Churchill Livingstone Inc., NY.

Penzes, T., Blazso, G., Aigner, Z., Falkay, G. and Eros, I. (2005). Topical absorption of piroxicam from organogels--in vitro and in vivo correlations. Int. J. Pharm. 298, 47-54.

Riviere, J. E. and Heit, M. C. (1997). Electrically-assisted transdermal drug delivery. Pharm. Res. 14, 687-697.

Rolf, C., Engstrom, B., Beauchard, C., Jacobs, L. D. and Le Liboux, A. (1999). Intra-articular absorption and distribution of ketoprofen after topical plaster application and oral intake in 100 patients undergoing knee arthroscopy. Rheumatology (Oxford). 38, 564-567.

Romsing, J., Mysager, S., Vilmann, P., Sonne, J., Larsen, N. E. and Stergaard, D. (2001). Postoperative analgesia is not different after local vs systemic administration of meloxicam in patients undergoing inguinal hernia repair. Can. J. Anesth. 48, 978-984.
Rosim, G. C., Barbieri, C. H., Lancas, F. M. and Mazzer, N. (2005). Diclofenac phonophoresis in human volunteers. Ultrasound. Med. Biol. 31, 337-343.

Schrier, D. J., Moniot, S., Gluckman, M. I. and Gibertson, R. B. (1987). The topical anti-inflammatory effects of a topical preparation of meclofenamic acid on carrageenan-induced footpad swelling in mice. J. Pharm. Pharmacol. 39, 57.

Sekiguchi, M., Shirasaka, M., Konno, S. and Kikuchi, S. (2008). Analgesic effect of percutaneously absorbed non-steroidal anti-inflammatory drugs: an experimental study in a rat acute inflammation model. BMC Musculoskelet Disord. 9, 15

Tyle, P. and Agrawala, P. (1989). Drug delivery by phonophoresis. Pharm. Res. 6, 355-359.

Todd, P. A. and Sorkin, E. M. (1988). Diclofenac sodium. A reappraisal of its pharmacodynamic and pharmacokinetic properties, and therapeutic efficacy. Drugs 35, 244-285.

Tyle, P. and Agrawala, P. (1989). Drug delivery by phonophoresis. Pharm. Res. 6, 355-361.

Vane, J. R. (1971). Inhibition of prostaglandin synthesis as a mechanism of action for aspirin-like drugs. Nat. New. Biol. 231, 232-235.

Vlak, T. (1999). Comparative study of the efficacy of ultrasound and sonophoresis in the treatment of painful shoulder syndrome. Reumatizam. 46, 5-11.

Wells, P. N. (1977). Ultrasonics in medicine and biology. Phys. Med. Biol. 22, 629-669.

Xin, B., Yokoyama, Y., Shigeto, T., Futagami, M. and Mizunuma, H. (2007). Inhibitory effect of meloxicam, a selective cyclooxygenase-2 inhibitor, and ciglitazone, a peroxisome proliferator-activated receptor gamma ligand, on the growth of human ovarian cancers. Cancer 110, 791-800.

Yang, J. H., Kim, D. K., Kim, T. Y., Kim, G. Y. and Shin, S. C. (2005). Anti-inflammatory effects by transdermal application of triamcinolone acetonide gel using phonophoresis in rats. Int. J. Pharm. 302, 39-46.

Yang, J. H., Kim, T. Y., Lee, J. H., Yoon, S. W., Yang, K. H. and Shin, S. C. (2008). Anti-hyperalgesic and anti-inflammatory effects of ketorolac tromethamine gel using pulsed ultrasound in inflamed rats. Arch. Pharm. Res. 31, 511-517.

Yocum, D., Fleischmann, R., Dalgin, P., Caldwell, J., Hall, D. and Roszko, P. (2000). Safety and efficacy of meloxicam in the treatment of osteoarthritis: a 12-week, double-blind, multiple-dose, placebo-controlled trial. The meloxicam osteoarthritis investigators. Arch. Intern. Med. 160, 2947-2954. 